\title{
Extrapolating EISCAT Pedersen conductances to other parts of the sky using ground-based TV auroral images
}

\author{
M. J. Kosch, T. Hagfors, K. Schlegel \\ Max-Planck-Institut für Aeronomie, Postfach 20, D-37189 Katlenburg-Lindau, Germany
}

Received: 25 June 1997 / Revised: 19 January 1998 / Accepted: 21 January 1998

\begin{abstract}
Ionospheric conductivity is not very easily measured directly. Incoherent scatter radars perhaps offer the best method but can only measure at one point in the sky at any one time and are limited in their time resolution. Statistical models of average conductivity are available but these may not be applied to individual case studies such as substorms. There are many instances where a real-time estimate of ionospheric conductivity over a large field-of-view is highly desirable at a high temporal and spatial resolution. We show that it is possible to make a reasonable estimate of the noctural height-integrated Pedersen conductivity, or conductance, with a single all-sky TV camera operating at $557.7 \mathrm{~nm}$. This is not so in the case of the Hall conductance where at least two auroral wavelengths should be imaged in order to estimate additionally the energy of the precipitating particles.
\end{abstract}

Key words. Atmospheric composition and structure (airglow and aurora) · Magnetospheric physics (auroral phenomena, instruments and techniques)

\section{Introduction}

The STARE bistatic coherent-backscatter radar system (Greenwald et al., 1978) has been employed to estimate the average statistical spatial distribution of horizontal E-region electric fields over Scandinavia. From this, in conjunction with the ionospheric Pedersen conductivity, Joule heating (Kosch and Nielsen, 1995) and Birkeland current (Nielsen, private communication) average global estimates have been made. Although STARE produces reasonable estimates of the ionospheric electric fields (Nielsen and Schlegel, 1983, 1985) with high temporal

Correspondence to: M. J. Kosch (typically $20 \mathrm{~s}$ ) and spatial (typically $20 \times 20 \mathrm{~km}$ ) resolution over a large field-of-view $(520 \times 520 \mathrm{~km})$, it cannot provide the ionospheric conductivity. For this reason, it was necessary to use a global, statistically averaged, model of ionospheric conductivity. Several such empirical models are available for height-integrated conductivities, or conductances (e.g. Wallis and Budzinski, 1981; Spiro et al., 1982; Hardy et al., 1987). All such models are essentially static except for some fairly crude range steps in $A e$ or $K_{p}$. Especially for nocturnal conductivities at high latitudes, local perturbations such as auroral precipitation may dominate, making statistical conductivity models inappropriate for case studies. Brekke and Moen (1993) point out that an urgent need exists to produce detailed global conductivity maps for the study of auroral substorm phenomena.

To study real-time Joule heating and Birkeland currents during substorms, it is necessary that an estimate of the Pedersen conductance be made within the STARE field-of-view at the same temporal and spatial resolution as the radar. The EISCAT radar (Folkestad et al., 1983; Rishbeth and van Eyken, 1993), which is conveniently located within STARE's field-ofview, can make height-resolved estimates of both the ionospheric Hall and Pedersen conductivities (e.g. Brekke and Hall, 1988; Schlegel, 1988). However good the measurement is though, it can only be made at a single point in the sky at any one time with a limited time resolution, hence the need for a method of extrapolation to a wider field-of-view. Although the aurora can fluctuate on a time scale of seconds or less, the EISCAT data is normally integrated for $2 \mathrm{~min}$ in order to achieve an acceptable signal-to-noise ratio.

It is well known that nocturnal ionospheric conductances can be inferred from optical data. Robinson et al. (1989) used satellite auroral images although these are rarely useful for real-time studies. Mende et al. (1984) used ground-based meridian-scanning photometers but they have a very small field-of-view. They suggested that monochrome auroral imagers could be used to extend the field-of-view to two dimensions. Kaila et al. (1993) 
have demonstrated that it is possible to use white-light TV images to expand the field-of-view of photometer measurements, thereby enabling them to estimate the auroral energy flux in a large field-of-view and hence obtain total electron density and, in principle, conductance. Rothwell et al. (1992) reported a linear dependence between variations in the peak value of electron density profile, from EISCAT, and auroral luminosity. It has been demonstrated that the vertical luminosity profile of an auroral form can sometimes be converted into ionisation rate and electron density profiles in agreement with EISCAT (Kaila, private communication). Although an incoherent scatter radar can make a much more accurate estimate of conductance, the same measurement by optical means is much easier to make on a large spatial scale with high time-resolution (Mende et al., 1984). In the present work, we seek to make conductance measurements from a single auroral imager with a large field-of-view, with high temporal and spatial resolution, at a single convenient wavelength.

\section{Methods and instrumentation}

The theoretical equations expressing ionospheric conductivity are well established (e.g. Brekke and Moen, 1993, and references therein) and will not be reiterated here. The application of these equations to incoherent scatter radar data is also well established (e.g. Schlegel, 1988). Instead, we derive the relationship between conductance and optical intensity. The continuity equation for the E-region electron densities $\left(N_{e}\right)$ measured at a given altitude is given by (Brekke et al., 1989a):

$\frac{\mathrm{d} N_{e}}{\mathrm{~d} t}=Q_{p}+Q_{t}-\alpha N_{e}^{2}-\operatorname{div}\left(N_{e} \cdot \bar{v}\right)$

where $Q_{\mathrm{p}}$ is the ion production rate due to auroral precipitation, $Q_{\mathrm{s}}$ is the ion production rate due to solar radiation, $\alpha$ is the height dependent effective recombination coefficient and $\bar{v}$ is the electron bulk velocity. Rearranging, we have:

$\alpha N_{e}^{2}=Q_{p}+Q_{s}-\frac{\mathrm{d} N_{e}}{\mathrm{~d} t}-\operatorname{div}\left(N_{e} \cdot \bar{v}\right)$.

Assuming night-time, steady-state conditions and that the last term $\left(N_{e} \cdot \bar{v}\right)$ can be neglected when compared to $\alpha N_{e}^{2}$ (Brekke et al., 1989a), the equation simply becomes: $\alpha N_{e}^{2}=Q_{p}$.

Auroral particles bombarding the atmosphere will lose energy at the rate of about $35 \mathrm{eV}$ per ion pair formed (Rees and Luckey, 1974). Upon recombination, only a small fraction $(\approx 4 \%)$ of the energy thus deposited results in spectroscopic emissions observable from the ground (Rees and Luckey, 1974). However, for a given precipitation rate by particles of a certain energy, it is reasonable to expect the production of auroral photons to remain constant. To first order, we can assume that auroral optical intensity $(I)$ is proportional to $Q_{p}$. That conductivity $(C)$ is proportional to $N_{e}$ is well established (e.g. Egeland et al., 1973). Hence, for conductivity variations associated with particle precipitation we have:

$C \propto \sqrt{I}$.

Still assuming night-time, it is worth noting the likely consequence of a breakdown of the other assumptions. Using Eq. (2) with $I \propto Q_{p}$ and $C \propto N_{e}$ we have:

$C \propto \sqrt{I-\frac{\mathrm{d} N_{e}}{\mathrm{~d} t}-\operatorname{div}\left(N_{e} \cdot \bar{v}\right)}$.

Clearly, during non-steady-state conditions and any significant divergence associated with the electron bulk velocity, the conductivity will be less than that expected from Eq. (4).

A single auroral imager cannot make an unambiguous height determination of the aurora. Since we can only measure $\Sigma I$, which is a column integral, altitude dependent effects are neglected. Of course, this is not strictly correct as the ion-neutral collision frequency, which appears as terms in the conductivity equations (e.g. Egeland et al., 1973), increases with decreasing altitude. It is largely for this reason that Pedersen conductivity peaks at a higher altitude than Hall conductivity, depending on the precipitating particle energy (Robinson et al., 1989; Vickrey et al., 1981). EISCAT data show that main contribution to the Pedersen and Hall conductivities from precipitating particles comes from $120-150 \mathrm{~km}$ and $80-130 \mathrm{~km}$ altitudes, respectively (Senior, 1991). High energy particles penetrate to lower altitudes in the atmosphere thereby contributing to both conductivities, whereas low energy particles do not penetrate so deeply and hence mostly only contribute to the Pedersen component. As a result of this, the Hall and Pedersen conductivities are strongly and weakly, respectively, energy dependent. Vickrey et al. (1981) show that the Hall and Pedersen conductances vary by factors of about 10 and 2, respectively, for an order of magnitude change in characteristic energy of the precipitating particles. Of course, both conductances are equally flux dependent. Several empirical formula have been produced from optical data for conductance (Hardy et al., 1987; Robinson et al., 1989; Spiro et al., 1982). They all show a stronger dependence on energy for the Hall component than for the Pedersen component.

The ratio of Hall to Pedersen conductance is widely used as an indicator of the energy of precipitating electrons in the auroral zone (Brekke et al., 1989a and references therein; Brekke et al., 1989b). An estimate of the energy can also be made spectroscopically if at least two wavelengths are measured simultaneously (Rees and Luckey, 1974). For this purpose, the ratio 630.0/427.8 appears to be the best although other auroral emissions also suffice. Comparisons between photometric and incoherent scatter radar techniques for estimating the energy of precipitating particles show good agreement (Wickwar et al., 1975; Vondrak and Sears, 1978).

The Digital All-Sky Imager (DASI) (Kosch et al., 1998) has been designed to complement STARE, and include EISCAT, for studies of the aurora. The detector consists of a monochrome low-light-level TV camera 
fitted with an all-sky lens. An interference filter at $557.7 \mathrm{~nm}$ selects the strongest auroral emission. The camera has been calibrated, as well as flat-field corrected, in Rayleighs at $557.7 \mathrm{~nm}$ using a radioactive light source. The Rayleigh is a non-SI unit still commonly used in auroral physics: By definition, 1 Rayleigh $=10^{6}$ photons $/ \mathrm{cm}^{2} / \mathrm{s}$, which is the number of photons emitted per second in $4 \pi$ steradian as viewed through a one square centimetre column integrated vertically through the emitting region at the selected wavelength (Hunten et al., 1956).

DASI is fully unmanned and is automatically operated by a PC for all dark and moon-free periods. The TV images are digitially integrated in real-time with a user-defined temporal resolution (normally $10 \mathrm{~s}$ ), transformed from all-sky format into any spatial grid (currently the field of view of STARE at $10 \times 10 \mathrm{~km}$ resolution for $100 \mathrm{~km}$ altitude) and saved to disk for later analysis. The spatial orientation of the camera and lens is fixed through star observations. The frequent gain changes necessary are computer controlled and recorded by the software, thus permitting each processed image to be recalled in Rayleighs at $557.7 \mathrm{~nm}$. DASI has been located at Skibotn, Norway $\left(69.35^{\circ} \mathrm{N}\right.$, $\left.20.36^{\circ} \mathrm{E}\right)$, which is near the centre of STARE's field-of view and about $50 \mathrm{~km}$ from EISCAT $\left(69.59^{\circ} \mathrm{N}\right.$, $\left.19.23^{\circ} \mathrm{E}\right)$ because of the superior local night-sky viewing conditions. Figure 1 shows the STARE field-of-view $\left(67.6-72.6^{\circ} \mathrm{N}, 13.5-26.0^{\circ} \mathrm{E}\right)$ at an assumed altitude of $100 \mathrm{~km}$ as seen by DASI from Skibotn in geographic coordinates. The position of EISCAT's beam (cross), when magnetic field aligned, is also shown for the same altitude.

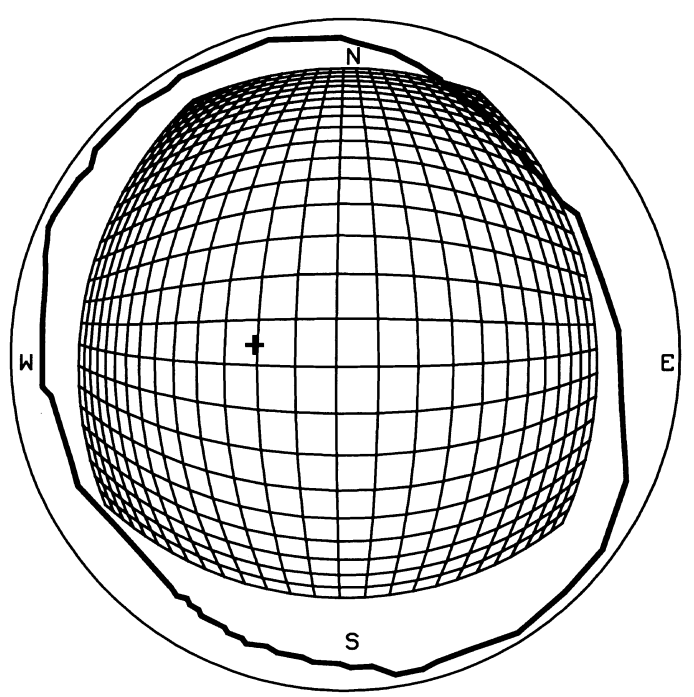

Fig. 1. The STARE field of view $\left(67.6-72.6^{\circ} \mathrm{N}, 13.5-26.0^{\circ} \mathrm{E}\right)$ at an assumed altitude of $100 \mathrm{~km}$ as seen by DASI through an all-sky lens from Skibotn $\left(69.35^{\circ} \mathrm{N}, 20.36^{\circ} \mathrm{E}\right)$ in geographic coordinates. The data grid is shown at the lower $20 \times 20 \mathrm{~km}$ resolution only for clarity. The position of EISCAT's beam (cross) when magnetic field aligned is also shown for the same altitude. The circle is the ideal horizon whilst the heavy irregular circle is the actual horizon
DASI is well suited to make Pedersen conductance estimates everywhere within STARE's field-of-view as it is calibrated, automatically makes column integrals of total optical intensity $(\Sigma I)$, has a better temporal and spatial resolution than STARE and includes EISCAT.

\section{Data analysis and discussion}

EISCAT is operated for only about $2000 \mathrm{~h}$ per year. DASI operates for about $1500 \mathrm{~h}$ per auroral season but only about $100-200 \mathrm{~h}$ of useful data result due to the frequently heavy cloud cover over northern Scandinavia. Hence, it is not always easy to find suitable simultaneous data sets, especially of longer runs. For this study we have used data from the nights of 28 February to 2 March $1995\left(K_{p}=4-6^{-}\right)$, the night of 28-29 March $1995\left(K_{p}=2^{-}-3^{+}\right)$as well as the night of 13-14 February $1996\left(K_{p}=3^{-} 4^{-}\right)$. The four recording nights represent a good spread of low to moderate geomagnetic activity $\left(K_{p}=2^{-}-6^{-}\right)$. On all these occasions, EISCAT was operated in the CP1 mode whereby the transmitter beam is kept continuously pointing along the magnetic field line direction. In this mode, conductivity estimates are made between 87 and $269 \mathrm{~km}$ altitude every $2 \mathrm{~min}$ (Schlegel, 1988). The total data set consists of 794 points with 316 pre- and 478 postmagnetic midnight $(\approx 21: 30 \mathrm{UT})$. Calibrated DASI data was extracted from the $10 \times 10 \mathrm{~km}$ spatial cell that overlaid the EISCAT beam. The $10 \mathrm{~s}$ optical measurements were averaged down to 2 min intervals which exactly correspond to the EISCAT data.

Figure 2 shows scatter plots of conductance, from EISCAT, versus auroral optical intensity, from DASI, using the entire data set. Figure $2 \mathrm{~A}$ shows the Hall component, Fig. 2B the Pedersen component and Fig. 2C the Hall to Pedersen ratio. Figure 2B also shows the least-squares fit of Eq. (4) to the data. The data has been divided into two ranges with solid squares for $K_{p}<4$ and crosses for $K_{p} \geq 4$. For $K_{p}<4$ the auroral intensity is restricted to approximately 500-3500 Rayleighs whereas for $K_{p} \geq 4$ all auroral intensities are represented in the data set. The lack of data below about 500 Rayleighs is partly due to the approaching sensitivity limit of the imager (Kosch et al., 1998).

It is clear that the Hall to Pedersen conductance ratio varies significantly for any auroral intensity. Since this ratio is an indicator of the energy of precipitating particles whereas auroral intensity relates directly to the flux of precipitating particles, Fig. 2C implies a significant spread in the energy of the precipitating particles which does not relate simply to the flux of auroral photons. This is reflected as a large spread in the scatter plot of Fig. 2A since the Hall conductivity is sensitive to the energy of precipitating particles. Although Hall conductance bears some relationship to auroral intensity, we are unable to make a meaningful curve fit using optical data at one wavelength alone. At least two auroral wavelengths need to be imaged in order to make an estimate of the energy of the precipitating particles. 

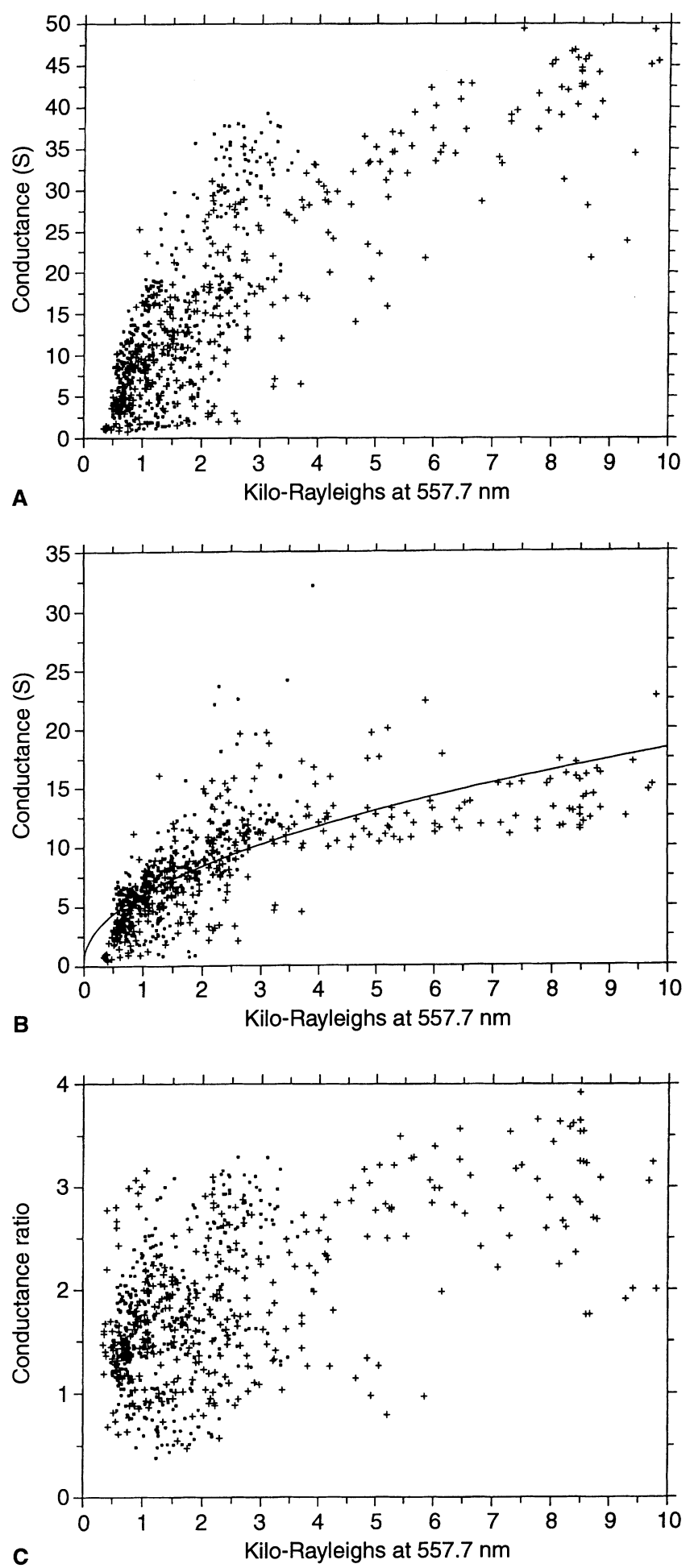

Fig. 2A-C. Scatter plots of conductance versus optical intensity at $557.7 \mathrm{~nm}$. A is the Hall component, B the Pedersen component with least-squares curve fit of Eq. (4) and $\mathbf{C}$ the Hall to Pedersen ratio. Solid squares correspond to data for $K_{p}<4$ whereas crosses are for $K_{p} \geq 4$

The Pedersen conductance, shown in Fig. 2B, does relate to auroral intensity rather well although the spread in the scatter plot is probably, at least partly, also due to the energy of the precipitating particles. Ignoring energy, the least squares fit of Eq. (4) gives:
$P=0.34+0.18 \sqrt{\Sigma I}$

where $P$ is Pedersen conductance (Siemens) and $\Sigma I$ is Rayleighs at $557.7 \mathrm{~nm}$. Equation (6) predicts a minimum Pedersen conductance of 0.34 for no auroral precipitation. During night-time, a small residual conductivity is expected from cosmic and galactic EUV radiation and is consistent with the value of 0.2 adopted by Senior (1991). Above about 4000 Rayleighs, the fit appears to be consistently too high. This intensity range results only from data taken at higher geomagnetic activity $\left(K_{p} \geq 4\right)$. Hence, it is not unexpected that the assumptions of steady state and negligible divergence used in deriving Eqs. (3) and (4) may be violated. Indeed, it is expected, from Eqs. (5), that the measured conductance should be less than predicted for nonsteady state conditions, which is consistent with our results.

The standard deviation from the mean is shown in Table 1 for different intensity ranges. The accuracy of the curve fit is approximately $30-45 \%$ for lower auroral intensities ( $<4000$ Rayleighs) improving to around 20$25 \%$ for higher intensities. The lower accuracy at lower intensities partially reflects the increasing noise in the TV image as the gain of the camera is increased. For low to moderate auroral intensities ( $<4000$ Rayleighs), Eq. (6) is a good representation of Pedersen conductance. For high auroral intensities ( $>4000$ Rayleighs) additional effects, which have not been accounted for, results in Eq. (6) being an overestimate. Figure 3 shows an example of using a DASI auroral image in conjunction with Eq. (6) to achieve an instantaneous map of Pedersen conductance throughout STARE's field of view.

The analysis was repeated using pre- and postmagnetic midnight data only to check for any systematic differences. The pre-midnight fit gives $P=0.47+$ $0.17 \sqrt{\Sigma I}$ and post-midnight the fit gives $P=0.13+$ $0.19 \sqrt{\Sigma I}$. Given the achievable accuracy, there is no significant difference in the fit for pre- or post- magnetic midnight Pedersen conductances.

Table 1. The mean and standard deviation of Pedersen conductance corresponding to the curve fit of Eq. (6), shown in the scatter plot of Fig. 2B, for different auroral intensity ranges

\begin{tabular}{lll}
\hline $\begin{array}{l}\text { Intensity range } \\
\text { (kilo-Rayleighs) }\end{array}$ & Mean & $\begin{array}{l}\text { Standard } \\
\text { deviation }\end{array}$ \\
\hline $0.5-1.0$ & 5.1 & 1.7 \\
$1.0-1.5$ & 6.6 & 2.1 \\
$1.5-2.0$ & 7.8 & 2.6 \\
$2.0-2.5$ & 8.8 & 3.6 \\
$2.5-3.0$ & 9.7 & 3.9 \\
$3.0-3.5$ & 10.5 & 4.4 \\
$3.5-4.5$ & 11.7 & 5.2 \\
$4.5-5.5$ & 13.1 & 3.1 \\
$5.5-6.5$ & 14.3 & 3.3 \\
$6.5-7.5$ & 15.4 & 2.6 \\
$7.5-8.5$ & 16.4 & 3.5 \\
$8.5-9.5$ & 17.4 & 3.3 \\
\hline
\end{tabular}




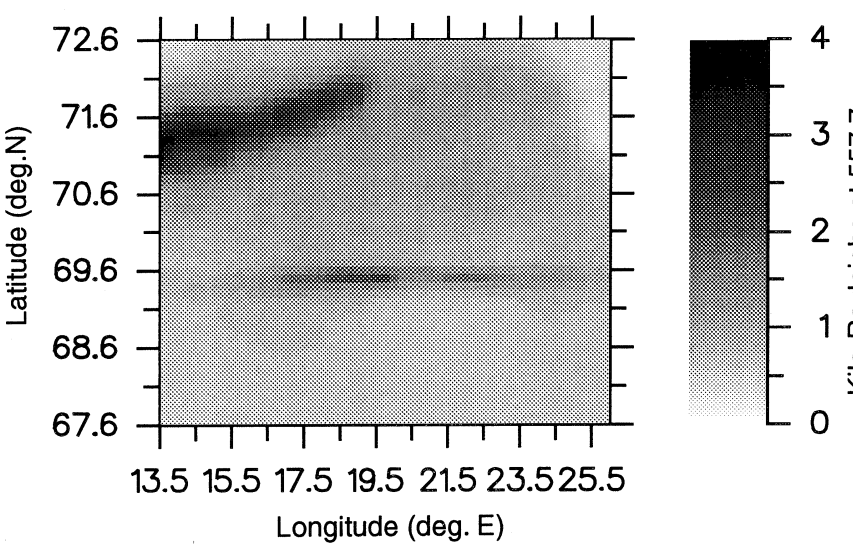

Fig. 3. An example of an auroral image taken by DASI calibrated in Rayleighs and the computed distribution of Pedersen conductance resulting from auroral precipitation. The left panel shows a $10 \mathrm{~s}$

There are several sources of potential uncertainty in this study including:

1. The steady state assumption may be violated. This is clearly the case during substorms.

2. Assumptions are made in producing the EISCAT conductivity estimate, for example, the ion-neutral collision frequency is taken from a model.

3. The accuracy of the camera intensity calibration depends on the confidence one has in the reference source. Radioactive light sources make relatively good standards and age fairly slowly. Low-light-level TV cameras do not lend themselves well to an absolute intensity calibration due to the inherently noisy nature of their image intensifiers which also suffer from degradation with time. Regular recalibration is necessary.

4. Some optical intensity dependence at $557.7 \mathrm{~nm}$ on the precipitating particle energy spectrum can be expected. 5. Some geometric ambiguity due to the camera not being exactly co-located with EISCAT is expected. Also, the selected $10 \times 10 \mathrm{~km}$ optical data cell does not exactly correspond to the radar beam only which has a $2 \mathrm{~km}$ diameter at $100 \mathrm{~km}$ altitude due to beam divergence.

6. When using ground-based optical images, perspective effects due to the vertical extent of the aurora will give an increasingly poor result the further one deviates from the local magnetic field line. In particular, aurora in one data cell but at a much greater height than $100 \mathrm{~km}$ may map into an adjacent data cell. This problem can not be avoided with a single imaging system. Similarily, when viewing an auroral arc not in the local magnetic zenith, the total column emission, and hence conductance, may be under- or overestimated depending on the geometry.

We have tried to fit EISCAT conductances using white light optical data from DASI for the nights of 18-20 October 1993. However, this proved to be impossible because the spread in the scatter plot was much too large. At this time, it is not known if using any wavelength other than $557.7 \mathrm{~nm}$ will give a better result.
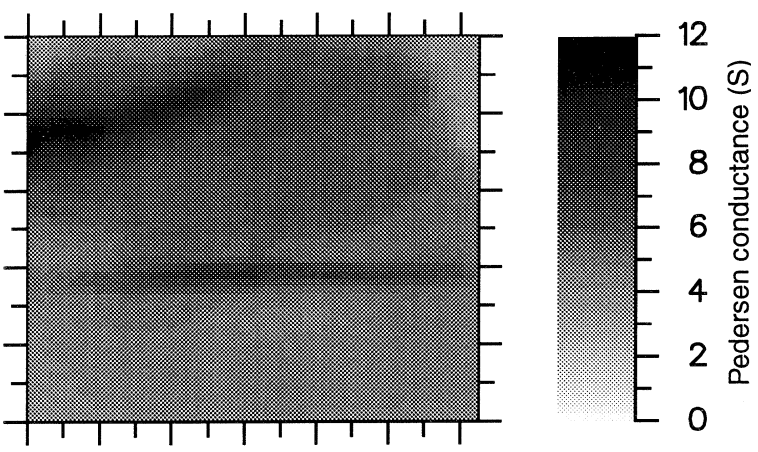

\subsection{5 .517 .519 .521 .523 .525 .5 \\ Longitude (deg. E)}

average of the auroral intensity taken at 18:47:30 UT on 13 February 1996. The right panel shows conductance throughout the STARE field of view as computed from Eq. (6)

\section{Conclusion}

We have shown that it is reasonable to use all-sky auroral images at $557.7 \mathrm{~nm}$ for estimating nocturnal ionospheric Pedersen conductances. No other additional observations are needed. The equivalent Hall conductances cannot be reasonably estimated from a single wavelength optical image. This is due to the greater dependence of the Hall conductivity on the energy of the precipitating particles. In order to use optical auroral images for estimating ionospheric Hall conductances, at least two wavelengths would have to be imaged simultaneously. A second all-sky imager, to operate at $630.0 \mathrm{~nm}$, is already under construction.

All-sky auroral imagers are relatively simple and cheap to build and offer a very high temporal and spatial resolution over a large field of view. Typical CCD technology can easily image the aurora at 557.7 or $630.0 \mathrm{~nm}$. Given the large numbers of all-sky cameras available at northern high-latitudes, it should be possible to produce large-scale real-time maps of nocturnal conductance. The use of tomographic reconstruction from multiple optical observing sites would ultimately permit the vertical profile of ionospheric conductivity to be determined also.

Combining data from the STARE and DASI experiments will permit real-time computation of highlatitude ionospheric Joule heating, as well as Birkeland current estimates, with high time and spatial resolution. Such studies are planned.

Acknowledgements. Topical Editor D. Alcaydé thanks J. Lilensten and K. Kaila for their help in evaluating this paper.

\section{References}

Brekke, A. and C. Hall, Auroral ionospheric quiet summer time conductances, Annales Geophysicae, 6, 361-376, 1988.

Brekke, A., and J. Moen, Observations of high latitude ionospheric conductances, J. Atmos. Terr. Phys., 55, 1493-1512, 1993.

Brekke, A., C. Hall, and T. L. Hansen, Auroral ionospheric conductances during disturbed conditions, Annales Geophysicae, 7, 269-280, 1989a. 
Brekke, A., C. Hall, and T. L. Hansen, EISCAT studies of the auroral ionosphere conductances, Adv. Space Res. 9, 35-38, 1989 b.

Egeland, A., Ø. Holter, and A. Omholt (Eds), Cosmical geophysics, Universitetsforlaget, Oslo-Bergen-Tromsø, p. 184, 1973.

Folkestad, K., T. Hagfors, and S. Westerlund, EISCAT: an updated description of technical characteristics and operational details, Radio Sci., 18, 867-879, 1983.

Greenwald, R. A., W. Weiss, E. Nielsen, and N. R. Thomson, STARE: a new radar auroral backscatter experiment in northern Scandinavia, Radio Sci., 13, 1021-1039, 1978.

Hardy, D. A., M. S. Gussenhoven, and R. Raistrick, Statistical and functional representations of the pattern of auroral energy flux, number flux, and conductivity, J. Geophys. Res., 92, 1227512294, 1987.

Hunten, D. M., F. E. Roach, and J. W. Chamberlain, A photometric unit for the airglow and aurora, J. Atmos. Terr. Phys., 8, 345346, 1956.

Kaila, K., R. Rasinkangas, and J. Kangas, Photometric calibration of auroral TV pictures during a pulsating patch event, Annales Geophysicae, 11, 790-796, 1993.

Kosch, M. J., and E. Nielsen, Coherent radar estimates of average high-latitude ionospheric Joule heating, J. Geophys. Res. 100, 12201-12215, 1995.

Kosch, M. J., T. Hagfors, and E. Nielsen, A new digital all-sky imager experiment for optical auroral studies in conjunction with the Scandinavian twin auroral radar experiment, Rev. Sci. Inst., 69, 578-584, 1998.

Mende, S. B., R. H. Eather, M. H. Rees, R. R. Vondrak, and R. M. Robinson, Optical mapping of ionospheric conductances, $J$. Geophys. Res., 89, 1755-1763, 1984.

Nielsen, E., and K. Schlegel, A first comparison of STARE and EISCAT electron drift velocities measurements, J. Geophys. Res. 88, 5745-5750, 1983.

Nielsen, E., and K. Schlegel, Coherent radar Doppler measurments and their relationship to the ionsopheric electron drift velocity, J. Geophys. Res., 90, 3498-3504, 1985.
Rees, M. H., and D. Luckey, Auroral electron energy derived from ratio of spectroscopic emissions, 1. Model computations, J. Geophys. Res., 79, 5181-5186, 1974.

Rishbeth, H., and A. P van Eyken, EISCAT: early history and the first ten years of operation, J. Atmos. Terr. Phys., 55, 525-542, 1993.

Robinson, R., R. Vondrak, J. Craven, L. Frank, and K. Miller, A comparison of ionospheric conductances and auroral luminosities observed simultaneously with the Chatanika Radar and the DE1 Auroral Imagers J. Geophys. Res., 94, 5382-5396, 1989.

Rothwell, P., S. Chernous, K. Schlegel, R. Pipenbrink, YU. Novikov, and D. Zaitsev, Possibilities of ionospheric electron density diagnostic by optical TV measurements. Proce 19th Annual European meeting on Atmospheric Studies by Optical Methods, 10-14 August, Kiruna. IRF Scientific Report 209, Ed. A. Steen, 1992.

Schlegel, K., Auroral zone E-region conductivities during solar minimum derived from EISCAT data, Annales Geophysicae, 6, 129-138, 1988.

Senior, C., Solar and particle contributions to auroral heightintegrated conductivities from EISCAT data: a statistical study, Annales Geophysicae, 9, 449-460, 1991.

Spiro, R. W., P. H. Reiff, and L. J. Maher Jr., Precipitating electron energy flux and auroral zone conductances - an empirical model, J. Geophys. Res., 87, 8215-8227, 1982.

Vickrey, J. F., R. R. Vondrak, and S. J. Matthews, The diurnal and latitudinal variation of auroral zone ionospheric conductivity, J. Geophys. Res., 86, 65-75, 1981.

Vondrak, R. R., and, R. D. Sears, Comparison of incoherent scatter radar and photometric measurements of the energy distribution of auroral electrons, J. Geophys. Res., 83, 1665-1667, 1978.

Wallis, D. D., and E. E. Budzinski, Empirical models of height integrated conductivities, J. Geophys. Res., 86, 125-137, 1981.

Wickwar, V. B., M. J. Baron, and R. D. Sears, Auroral energy input from energetic electrons and Joule heating at chatanika, J. Geophys. Res., 80, 4364-4367, 1975. 